\title{
De la percepción del tiempo a la predicción del futuro, el progreso como fin
}

doi: $10.52749 /$ fh.v2i4.5

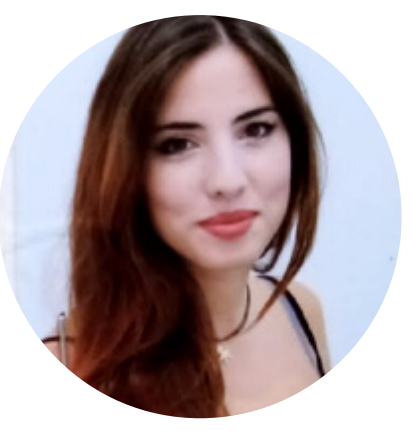

\section{CATERINA PRESUTTI (iD) https://orcid.org/0000-0003-0622-469X}

Magíster en Neurobiología y Bachiller en Biología por la Università La Sapienzade Roma, Italia.Actualmente estudia su PhD en Bioquímica, en MembraneEnzymology, del Departamento de Bioquímica de la Universidad de Groningen,Países Bajos, como parte del Marie Curie fellowship para el proyectoBiolnspireSensing (ITN-ETN).

presutti.1676243@studenti.uniroma1.it

Resumen. Sólo gracias a nuestra percepción intrínseca del tiempo hemos desarrollado la capacidad de extender nuestro pensamiento hacia el mañana. Este artículo es un breve viaje que parte de la filosofía Kantiana del tiempo con una breve parada en la neurobiología de la percepción del tiempo de Hermann Helmholtz y llega, por último, a la predicción del futuro aplicada a los tiempos modernos. Haber desarrollado a lo largo del tiempo la capacidad de anticipar los pensamientos y las acciones no es sólo un mecanismo evolutivo para ayudar al hombre como individuo, sino que, hoy en día, es cada vez más importante extenderlo en beneficio de todo el planeta. La anticipación de estrategias útiles se convierte en la base de los planes organizativos de las grandes empresas y corporaciones, que utilizan una peculiar táctica de predicción conocida como "Scenario Planning" para comprometerse en grandes objetivos que van desde la recuperación medioambiental y energética hasta la ayuda sanitaria y social en países en desarrollo como África. Sin embargo, los grandes planes sociales y medioambientales implican no sólo a las grandes empresas, sino sobre todo a las ciudades individuales, desarrollando lo que se conoce como "Smart Cities". La idea global de futuro y progreso, en conclusión, entra en sinergia con una antigua corriente filosófica y literaria del siglo XIX, el Positivismo. La fe del hombre en las fuerzas del progreso tecnológico y científico de entonces no parecerá tan lejana de la de hoy, que pone en manos de la tecnología, la ciencia y el hombre la esperanza de un mundo cada vez más sostenible e inteligente.

Palabras clave: Futuro, sociedad, tiempo, neurobiología, progreso, Smart Cities

No habría futuro si no existiera la percepción del tiempo. Immanuel Kant en su «Crítica de la razón pura» afirmó que el espacio y el tiempo son formas «puras», a priori, de la intuición, que existen antes de cualquier experiencia y que, gozando de esta naturaleza intuitiva, actúan como un sistema trascendental que conecta los datos fenomenales de la realidad entre sí (Abbagnano, 2017). En este sentido, nuestro propio pensamiento, desde el nacimiento, está inevitablemente impregnado por el concepto del tiempo, que, como él mismo define, es un sentido universal dado a todos los seres dotados de razón. Aunque el sentido del tiempo puede considerarse un acontecimiento de la conciencia, existe y es real. De hecho, la neurociencia ha demostrado que la percepción del tiempo es un mecanismo cerebral real. Ya en el año 1849, el fisió- logo médico alemán Hermann von Helmholtz, en sus estudios sobre la propagación de las señales nerviosas(Piccolino, 2009), abrió la puerta no sólo a una neurofisiología más consciente, sino también a una nueva disciplina filosófica centrada en el concepto de tiempo. No es casualidad, de hecho, que uno de los hitos de la literatura del siglo XX, "La Recherche du temps perdu" de Marcel Proust (18711922), naciera en ese periodo.

En sus experimentos, Hermann Helmholtz demostró que un estímulo eléctrico de corta duración generaba una contracción muscular que no sólo duraba más que la duración del estímulo, sino que además se producía con un cierto retraso (Piccolino, 2009). Definió este retraso como tiempo latente (temps perdu en francés), que hoy se entiende como el tiempo que tarda el cerebro en procesar un 
acontecimiento. La existencia de un "tiempo latente medible" demostró que la simultaneidad del estímulo y el efecto es una ilusión, y que es necesario un tiempo finito para el proceso de conducción nerviosa $y$, por tanto, para el procesamiento de un pensamiento o evento.

Por lo tanto, la idea de que el cerebro proporciona a los mecanismos nerviosos de la conciencia un sentido del tiempo no está demasiado lejos de la naturaleza intuitiva trascendental del concepto de tiempo descrito por Kant en 1789.

\section{La percepción del tiempo como herramienta para ver el futuro}

La existencia del "ayer" y del "mañana" que se repite periódicamente desde los primeros años de nuestra vida genera nuestra capacidad de percibir el futuro, esa dimensión espacio-temporal que aún no existe. La capacidad de planificar un futuro, anticipando los pensamientos $\mathrm{y}$, por tanto, las acciones, está de hecho en la base de nuestra evolución. Sin embargo, en el transcurso de la evolución resulta cada vez más crucial planificar un futuro que abarque no sólo al individuo, sino sobre todo a toda la humanidad y a todo el planeta. El verdadero progreso es ser capaz de prever un futuro que no excluya a nadie.

Uno de los ejemplos más modernos y evidentes de la ventaja evolutiva de la previsión proviene del concepto del "Scenario Planning", Planificación de escenarios. La "Planificación de escenarios" es un método de planificación estratégica basado en la previsión de posibles escenarios y que se utiliza para aplicar tácticas útiles a largo plazo. Es un método utilizado por varias organizaciones $y$ empresas, como DHL Express y Royal Dutch Shell, que tras la crisis energética de los años 80 fue una de las que más rápido se recuperó.

\section{El futuro como ética}

Hoy en día, cuando los problemas medioambientales y energéticos son cada vez más frecuentes, resulta especialmente importante dotar al futuro de un carácter ético y moral que sitúe no sólo al individuo sino a toda la comunidad en el centro de sus problemas. Los escenarios de futuro ayudan a tomar decisiones cruciales, especialmente en períodos de incertidumbre y transición como el actual. Los ejemplos de cómo la "Planificación de escenarios" está funcionando para el medio ambiente y el clima vienen, una vez más, de la colaboración de Shell con China (Lu \& Zhao, 2015). Un estudio conjunto entre Shell y el Centro de Investigación para el Desarrollo (DRC) del Consejo de Estado de China tiene como objetivo desarrollar el gas natural como una fuente de energía importante en China y aumentar la participación del gas en la mezcla de energía al 15\% para 2030 (desde el 5,8\% en el 2014) (Lu \& Zhao, 2015). El gas natural es un gas producido por la descomposición anaeróbica de la materia orgánica. En la naturaleza suele encontrarse en estado fósil, junto con el petróleo, el carbón o solo en los yacimientos de gas natural. Es la alternativa ideal al carbón, ya que es más limpio, más eficiente y más fácil de transportar y almacenar. De hecho, el desarrollo de escenarios futuros no sólo implica las posibles estrategias climáticas, sino también las destinadas a ayudar a la sociedad. Este es el caso de la colaboración de UNAIDS (Joint United Nations Programme on HIV and AIDS) con Shell para desarrollar escenarios (y luego planes de acción) sobre cómo podría propagarse el VIH/SIDA en las próximas dos décadas en países como África (Dougan, 2005), donde sólo en el 2009 se infectaron 1,8 millones de personas.

\section{Prever el futuro para potenciar el progreso}

Nuestro siglo se caracteriza por una enorme expansión urbana. A mediados de siglo podría haber más de 9.000 millones de personas en la Tierra y tres cuartas partes de ellas vivirán en ciudades. Uno de los mayores retos es construir ciudades más sostenibles que puedan gestionar las crecientes presiones sobre los recursos vitales de energía, agua y alimentos, ofreciendo al mismo tiempo formas más limpias de alimentar los hogares y los vehículos. La previsión y la planificación de los paisajes potenciales se convierten, en este contexto, en la base de la construcción de las llamadas "Smart Cities", ciudades inteligentes. Una ciudad inteligente es una ciudad que gestiona los recursos de forma inteligente, que aspira a ser económicamente sostenible y energéticamente autosuficiente, y que está atenta a la calidad de vida y a las necesidades de sus ciudadanos. Las características de una ciudad inteligente son múltiples. Estos van desde propiedades que benefician a los ciudadanos individuales hasta beneficios medioambientales. Los más evidentes son un alto nivel de conectividad de las calles, un transporte público eficiente, grandes espacios verdes, movilidad sostenible con coches híbridos, eléctricos y bicicletas, y un enfoque general en el uso de energías renovables. Como ejemplo, me gustaría mencionar la ciudad donde vivo, Groningen, la octava ciudad más grande de los Países Bajos, situada en el norte del país. Algunos de los proyectos de Smart City que la ciudad está llevando a cabo son 
el alumbrado público inteligente (nuevas farolas que se encienden y apagan en función de cuándo pasa alguien), los semáforos inteligentes (carriles bici equipados con semáforos, que dan prioridad a las bicicletas cuando llueve), la red eléctrica inteligente (un sistema de red inteligente, que minimiza las sobrecargas y las variaciones de la tensión eléctrica en torno a su valor nominal) y uno de los más famosos es el proyecto NortH2. El NortH2 es un proyecto europeo de hidrógeno verde entre Gasunie, Groningen Seaports y Shell Nederland. El objetivo es producir hidrógeno verde utilizando la electricidad renovable generada por un mega parque eólico marino, contribuyendo así a los objetivos del Acuerdo Holandés sobre el Clima.

\section{Conclusión}

Aunque estos nuevos enfoques que acabamos de mencionar parecen extremadamente modernos, la filosofía progresista, que constituye el trasfondo de todo esto, no está demasiado alejada de la corriente filosófica y literaria europea del siglo XIX conocida como Positivismo. El positivismo se originó en relación con el desarrollo económico industrial de la Europa de la época (Piromalli, 2007). En efecto, el desarrollo industrial de la época hizo que se confiara en las fuerzas del progreso tecnológico y científico del hombre. La razón daba confianza a la ciencia, que había descubierto nuevos principios y derivaba de ellos numerosas y variadas aplicaciones sociales, técnicas, industriales y prácticas. La indus-

\section{Referencias}

Abbagnano, N. (2017). Storia della filosofia (Vol. 3): La filosofia moderna e contemporanea: dal Romanticismo all'esistenzialismo. https://www.ibs.it/storia-della-filosofia-vol-3-libro-nicola-

abbagnano/e/9788851152543

Dougan, S. (2005). AIDS in Africa: three scenarios in 2025. Euro Surveillance: Bulletin Européen Sur Les Maladies Transmissibles European Communicable Disease Bulletin, 10(3), 2675. https://doi.org/10.2807/ESW.10.13.02675-EN/CITE/PLAINTEXT industria siderúrgica y química en Alemania, la industria textil y mecánica en Inglaterra, las aplicaciones del motor de combustión interna, los descubrimientos de laboratorio de Pasteur, Koch, Röntgen y los Curies tuvieron como base la ciencia positiva que estudia los hechos de acuerdo con leyes fundamentales destinadas a ver la racionalidad en la realidad, a aplicar los descubrimientos científicos para el progreso y la mejora continua de la sociedad (Piromalli, 2007). El culto desenfrenado a la ciencia y la tecnología se traduce así en una fe total en la evolución de las ciudades, la sociedad y la vida.

Auguste Comte, sociólogo y filósofo francés, considerado el fundador del Positivismo, escribió: "De la ciencia viene la predicción, de la predicción viene la acción" (Cours de philosophie positive, 18301842).

La afirmación de Comte en su antología resume en muy pocas palabras lo que se hace en realidad, por ejemplo, por la Planificación de Escenarios de diversas organizaciones/entidades/asociaciones. Entonces, todos estos años después, ¿cuánto hemos avanzado realmente desde el pasado? ¿Estamos readaptando lo que se planificó en el pasado a la actualidad? Es imposible dar una respuesta precisa a una pregunta tan vasta, pero lo que sí es cierto es que todo lo heredamos del pasado, y tal vez el futuro represente una hermosa esperanza de la que partir hoy para tener un mañana mejor.

Lu, J., \& Zhao, S. (2015). China's natural gas exploration and development strategies under the new normal. Natural Gas Industry B, 2(6), 473-480. https://doi.org/10.1016/J.NGIB.2015.12.001

Piccolino, M. (2009). A "lost time" between science and literature: the "temps perdu" from Hermann von Helmholtz to Marcel Proust. Audiological Medicine, 1(4), 261-270. https://doi.org/10.1080/16513860310023218

Piromalli, A. (2007). La storia della letteratura italiana scritta da Antonio Piromalli. http://www.storiadellaletteratura.it/main.php? cap $=18 \&$ par $=2$

\section{Cómo citar este artículo:}

Presutti, C. (2021). De la percepción del tiempo a la predicción del futuro, el progreso como fin. Futuro Hoy, 2(4), 28-30. https://doi.org/10.52749/fh.v2i4.5

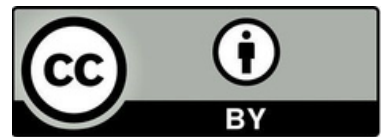

Esta obra está bajo licencia internacional Creative Commons 4.0 Reconocimiento 4.0. 